\title{
Robust Decoding of H.264 Encoded Video Transmitted over Wireless Channels
}

\author{
Galina Sabeva, Salma Ben Jamaa, Michel Kieffer, and Pierre Duhamel \\ LSS - CNRS - Supélec - Université Paris-Sud 11 \\ Plateau de Moulon, 91192 Gif-sur-Yvette, France
}

\begin{abstract}
Due to its high compression efficiency, the $\mathbf{H . 2 6 4}$ video coder is very sensitive to impairments due to transmission over noisy channels. Most error resilience/concealment techniques provided in the $\mathrm{H.264}$ standard were dealing with packet losses. In wireless environments, the proportion of corrupted packets (and thus considered as lost) may become very high. This paper shows that the $\mathbf{H . 2 6 4}$ decoder may be seen as a parity check decoder able to detect erroneous packets. Combined with soft estimation techniques, it allows to correct transmission errors and to reduce significantly the number of packets deemed lost. The proposed solution is compatible with the error-resilience features of $\mathbf{H . 2 6 4}$.
\end{abstract}

\section{INTRODUCTION}

The H.264 standard for video coding [1] achieves better video compression and improved network adaptation when compared to former standards [2]. These two advantages make H.264 efficient for wireless multimedia applications like video streaming, broadcasting or conversational services. Nevertheless, on the one hand, these applications require transmission over noisy packet-switched channels, where transmission errors range from single bit inversions to packet losses, and on the other hand, the high compression efficiency of the H.264 encoder makes it very sensitive to these transmission errors.

Usually, it is assumed that in the today transmission systems the multiplexing protocols include packet-loss and biterror detection capabilities [3]. For example, channel coding combined with ARQ mechanisms enable the vast majority of impairments to be detected and corrected, so that the decoder receives errorless packets. However, in applications such as video broadcasting or visiophony with mobile phones, ARQ is difficult to put at work due to delay constraints [3]. This is why H.264 includes error-resilience features at application level and error concealment capabilities [4]. Both were designed to make the H.264 decoder more robust to packet losses by preventing the propagation of errors due to lost data in the decoded video. They are applied in combination with channel coding techniques, thus increasing the required channel bandwidth.

However, when few errors remain in a packet after channel decoding, it would be much more efficient trying to correct these errors, instead of assuming that the packet is lost and using concealment techniques. This is the aim of joint sourcechannel decoding (JSCD) techniques, see, e.g., [5]-[10]. Video coders such as H.263 [11], MPEG4 [12] or H.264 generate bitstreams that have to comply to some syntax in order to be decodable (the purpose of the standard is to describe this syntax). Thus, not all successions of bits may be generated by a video coder resulting in some redundancy left in the bitstream. Using soft information provided by the channel decoder, JSCD exploits this redundancy to correct errors that have gone through the channel decoder, by providing bitstreams that are compliant with the encoder syntax. For H.263, MPEG4, or H.264 in the baseline profile, redundancy has been identified due to the structure of the variable-length codes used for entropy coding [5]-[7], to the fact that video is encoded macroblock by macroblock [8] and to the packetization of the encoded macroblocks [9].

The redundancy in bitstreams generated by $\mathrm{H} .264$ in the main or high profile, when using Context Adaptive Binary Arithmetic Coding (CABAC), is much more difficult to identify. Our purpose is to present a JSCD scheme adapted to this situation. It will be shown that the H.264 decoder may be used as an error-detecting code. Combined with a sequential channel decoder, transmission errors in received packets may be detected and corrected. This method does not introduce additional redundancy in the bitstream, in contrast with standard error correcting codes, as only the redundancy left by the H.264 encoder is involved. Moreover, the proposed technique remains compatible with the error-resilience features of H.264. A stronger cooperation between both techniques would bring further improvements.

Section II briefly recalls the structure of the H.264 encoder. A model of the wireless transmission and coding schemes is provided in Section III. The JSCD scheme involving the H.264 decoder combined with a sequential decoder is presented in Section IV. Experimental results are detailed in Section V.

\section{H.264 ENCODER}

The H.264 encoder is briefly described here. For more details, see [2]. Each picture of a video sequence is divided into macroblocks of $16 \times 16$ pixels. A group of macroblocks forms a slice of the picture. Each slice is encoded independently. For every macroblock $M_{n}$ of a slice, a prediction $P_{n}$ is evaluated and subtracted from the original macroblock $M_{n}$. The result is referred to as a difference macroblock $D_{n}$ and is passed through a video processing module, performing Discrete Cosine Transform (DCT), quantization and finally entropy coding. There are two types of prediction: intra and inter. For intra prediction, the encoder uses only information from the current slice. For inter prediction, data from the previously encoded pictures may also be used. 
The purpose of entropy coding is to compress data, according to the probabilities of the input symbols. H.264 involves two types of entropy coding: Context Adaptive Variable Length Coding (CAVLC) and Context-based Adaptive Binary Arithmetic Coding (CABAC).

As H.264 has been developed to be compatible with a large range of application contexts, several coding profiles have been defined, each profile consisting of a subset of coding tools meeting the coding requirements of a particular application. The three basic profiles are baseline, main and extended. Additionally, there are four versions of a so called high profile. Here, only profiles involving CABAC have been considered, but the results presented could readily be extended to profiles using CAVLC.

\section{A. $C A B A C$}

The CABAC [13] consists of three basic steps. As CABAC handles only binary data, the symbols obtained at the output of the video processing module are first binarized, i.e., transformed in bin strings (bits at the output of the binarization module are called bins). Then a context model is derived for every bin in the stream. The context model depends on the type of the binarized symbol, on the position of the bin in the stream, and on the previously encoded symbols. Arithmetic coding [14] is performed in a third step for every bin using probabilities deduced from the contexts. The context models and the probability counts are updated continuously.

By combining an adaptive binary encoding technique with context modeling, an improved compression efficiency is achieved especially in presence of non-stationary sources. For instance, CABAC results are up to $20 \%$ better than those obtained by the CAVLC [15]. However, when transmission errors occurs, CABAC makes the error detection and correction much more complicated [4], as a single bit error in a packet propagates in the remainder of this packet. Resynchronisation is much more difficult than with CAVLC.

\section{B. Network Abstraction Layer units}

H.264 consists of a Video Coding Layer (VCL) responsible for the video compression, and of a Network Abstraction Layer (NAL), which defines the interface between the VCL and the outside world. The NAL divides the bitstream into packets called NAL Units (NALUs) suitable for transmission over a wide variety of channels. Usually, each slice is put in a separate NALU.

When CABAC is used, the probability counts, the encoder state, and the contexts are initialized at the beginning of every NALU. This allows to send and decode NALUs more or less independently of the previous ones, and constitutes a first error-resilience tool. However, this reinitialization reduces the efficiency of CABAC and increases the size of the encoded bitstream.

\section{TRANSMISSION SCHEME}

A model of our wireless communications scheme is represented on Figure 1. It consists of an H.264 encoder (VCL and NAL), an H.264 decoder, and the transmission part itself. The output of the H.264 encoder is a sequence of NALUs. Let $\mathbf{x}_{1: N_{i}}^{i}=\left\{x_{1}^{i}, \ldots, x_{N_{i}}^{i}\right\}$ represent the succession of $N_{i}$ bits in the $i$-th NALU. The modulation and wireless part of the channel is modeled by a BPSK modulation and an Additive White Gaussian Noise (AWGN) channel. Let $\mathbf{y}_{1: N_{i}}^{i}=\left\{y_{1}^{i}, \ldots, y_{N_{i}}^{i}\right\}$ be the vector of $N_{i}$ real-valued channel outcomes, $N_{i}$ being known at the receiver.

Fig. 1. Transmission and coding scheme

Consider $X_{N_{i}}^{i}$, the set of all sequences of $N_{i}$ bits that could be generated by an H.264 encoder for the $i$-th NALU. The aim of the considered decoder is to provide a maximum-likelihood estimate $\widehat{\mathbf{x}}$ of $\mathbf{x}_{1: N_{i}}^{i}$ defined as

$$
\widehat{\mathbf{x}}=\arg \max _{\mathbf{x} \in X_{N_{i}}^{i}} p\left(\mathbf{y}_{1: N_{i}}^{i} \mid \mathbf{x}\right) .
$$

When the channel is memoryless and corrupted by additive white Gaussian noise (AWGN), (1) becomes

$$
\widehat{\mathbf{x}}=\arg \min _{\mathbf{x} \in X_{N_{i}}^{i}}\left\|\mathbf{y}_{1: N_{i}}^{i}-\mathbf{x}\right\|^{2} .
$$

Restricting the search space to sequences that are compatible with the syntax of H.264 allows to use the redundancy left by the video coder. However, if the maximization of $p\left(\mathbf{y}_{1: N_{i}}^{i} \mid \mathbf{x}\right)$ over all sequences of $N_{i}$ bits is straightforward for a memoryless channel, the maximisation constrained to all sequences of $X_{N_{i}}^{i}$ is much more complicated. An approximate solution is presented in the next section.

\section{JSCD OF H.264 NALUS}

At the input of the decoder, the received sequence $\mathbf{y}_{1: N_{i}}^{i}$ is a noisy version of the modulated NALU $\mathbf{x}_{1: N_{i}}^{i}$, see Figure 1. As the CABAC decoder, which is the first part of the H.264 decoder, accepts only binary data at its input, the channel decoding process has to feed it with hard decisions based on $\mathbf{y}_{1: N_{i}}^{i}$. It will be shown in Section IV-A that the H.264 decoder is able to detect erroneously estimated NALUs, provided that an estimation error results in an H.264 encoder syntax error. This property of the H.264 decoder allows to decide whether a sequence $\mathbf{x}$ belongs to $X_{N_{i}}^{i}$.

The H.264 decoder may thus be seen as a kind of paritycheck decoder. It is then combined with a sequential decoder presented in Section IV-B to provide an approximate solution for $(1)$.

\section{A. The H.264 decoder seen as a parity-check decoder}

The H.264 standard describes the way a bitstream generated by an H.264 encoder has to be organized. Restrictions are thus imposed to the bitstream. For a given NALU, all sequences of bits are not valid and a single bit error in a NALU may result in syntax errors that may be detected by the H.264 decoder.

Syntax errors may be detected in the NALU headers, e.g.,

- the value of the syntax element forbidden_zero_bit should be equal to 0 , 
- the value of the syntax element nal_unit_type, describing the type of NALU should range from 0 to 13,

- when the NALU contains critical parameters, such as Sequence Parameter Set or Picture Parameter Set [1], a zero_byte, placed in the NALU for checking its integrity, should be equal to 0x00,

A badly estimated NALU may also be evidenced when

- not exactly 99 macroblocks are decoded from the bitstream for a given picture in QCIF format,

- not exactly 396 macroblocks are decoded from the bitstream for a given picture in CIF format.

Various prediction modes are available to build $P_{n}$ for a given intra-encoded macroblock $M_{n}$. However, depending on the position of $M_{n}$ in the slice, not all prediction modes are allowed:

- when the macroblock $M_{n}$ is in the upper line of a slice, the syntax element prediction_mode should indicate that $P_{n}$ has to be evaluated from macroblocks on the left of $M_{n}$,

- when the macroblock $M_{n}$ is in the left column of a slice, the syntax element prediction_mode should indicate that $P_{n}$ has to be evaluated from macroblocks above $M_{n}$.

Other potential syntax errors, corresponding to redundancy left in the H.264 bitstream, may be evidenced but need a much more detailed presentation of the H.264 standard. When the H.264 syntax is satisfied for a candidate sequence of bits $\mathbf{x}$, nothing ensures that it corresponds to $\mathbf{x}_{1: N_{i}}^{i}$. However, if it is also the solution for $(1)$, it is the best estimate for $\mathbf{x}_{1: N_{i}}^{i}$ in the maximum-likelihood sense.

\section{B. Sequential estimation of the NALUs}

A possible way to solve (1) is to sort all sequences $\mathbf{x}$ of $N_{i}$ bits according to decreasing $p\left(\mathbf{y}_{1: N_{i}}^{i} \mid \mathbf{x}\right)$, in order to get $\mathbf{x}_{1}, \mathbf{x}_{2}, \mathbf{x}_{3} \ldots$ Then, $\widehat{\mathbf{x}}$ is chosen as $\mathbf{x}_{\bar{i}}$, where $\bar{i}$ is the smallest $i$ such that $\mathbf{x}_{i}$ satisfies the H.264 syntax compliance tests presented in Section IV-A. However, the combinatorial complexity of this approach makes it incompatible with realtime implementations.

The purpose of a sequential decoder [16] is to solve (1) with a manageable complexity. To the vector $\mathbf{y}_{1: N_{i}}^{i}$ of channel outcomes (see Figure 1) is assigned a decoding tree with $2^{N_{i}}$ paths corresponding to all possible bitstreams $\mathbf{x}$ of $N_{i}$ bits. Let $\mathbf{x}_{1: n}=\left\{x_{1}, \ldots, x_{n}\right\}$ be a path of $n$ bits of this decoding tree. The sequential decoder explores the decoding tree in order to find the best sequence according to a given metric, without examining too many paths. Here, the considered metric is derived from (1)

$$
\mathcal{M}\left(\mathbf{y}_{1: n}^{i}, \mathbf{x}_{1: n}\right)=-\log p\left(\mathbf{y}_{1: n}^{i} \mid \mathbf{x}_{1: n}\right) .
$$

For an AWGN channel, one may neglect the constant terms in (2) to get

$$
\mathcal{M}\left(\mathbf{y}_{1: n}^{i}, \mathbf{x}_{1: n}\right)=\sum_{k=1}^{n}\left(y_{k}^{i}-x_{k}\right)^{2},
$$

which has to be minimized in order to maximize $p\left(\mathbf{y}_{1: N_{i}}^{i} \mid \mathbf{x}\right)$.
The most popular sequential decoding algorithms are the stack algorithm (SA) and the M algorithm (MA) [16]. The MA performs a breadth first search, the breadth being $M$, and allows to control the decoding complexity, proportional to $M$, contrary to the SA. This is why it is considered here.

The steps for the MA are:

- Step 1. Initialize a list for the $M$ best paths with a single path containing the root of the decoding tree to which a null metric is assigned.

- Step 2. Extend all paths to the following branches, creating 2 new paths from each stored path.

- Step 3. Calculate the metric for each path and keep only the best $M$ paths according to the metric.

- Step 4. Stop if the $M$ maintained paths reach the desired depth, i.e., are $N_{i}$ bits long. Otherwise go to Step 2.

Note that the MA provides only an approximate solution to the problem of finding $\arg \max p\left(\mathbf{y}_{1: N_{i}}^{i} \mid \mathbf{x}\right)$. Moreover, when all $M$ candidates $\mathbf{x}_{1} \ldots \mathbf{x}_{M}$ provided by the MA do not satisfy the constraints imposed by the H.264 syntax, the NALU is assumed to be lost and standard error concealment techniques provided by H.264 are put at work.

\section{EXPERIMENTAL RESULTS AND DISCUSSION}

The simulations performed in this section are based on the transmission scheme presented in Section III. The 197 first frames of the sequence foreman.cif are encoded, modulated and transmitted to the decoder NALU by NALU through a noisy channel. Every altered NALU is fed to the MA in order to compute a set of $M$ estimates. These estimates are then provided to the H.264 syntax compliance checker, in decreasing likelihood order. If the current estimate of the NALU satisfies the H.264 syntax, it is provided to the H.264 decoder. If it is not the case, a syntax test is performed on the following estimate provided by the MA. When the whole set of $M$ estimates for the current NALU has been tested without giving any acceptable solution, the H.264 error concealment techniques are involved.

\begin{tabular}{l|l} 
Parameter & Value \\
\hline \hline Sequence & foreman.qcif $(100$ frames $)$ \\
\hline Entropic coding & CABAC \\
\hline Sequence Type & IBP \\
\hline QP for I Slices & 36 \\
\hline QP for P Slices & 34 \\
\hline QP for B Slices & 30 \\
\hline Force IDR Intra & enable \\
\hline NALU size limit & 120 or 160 bytes \\
\hline Picture structure & frame only
\end{tabular}

TABLE I

SOME ENCODING PARAMETERS USED FOR SIMULATIONS

The JM 10.2 implementation of H.264 [17] in its high profile has been used here. Some of the configuration parameters of the encoder are provided in Table I. Some of the error resilience techniques supported by H.264 [1] have been put at work, namely slice-structured coding and insertion of a fully 
intra-coded frame every 16 encoded frames. The size of a slice (and thus of a NALU) has been limited to 120 or 160 bytes. As mentioned in Section II-B, this size limitation introduces some encoder performance reduction. This fact is illustrated by Table II. However, with NALUs limited to 160 bytes, the size of the generated bitstream increases only by $6.77 \%$, which remains reasonable.

\begin{tabular}{c|c|c|c} 
NALU size & SNR-Y $(\mathrm{dB})$ & Bitstream size & Extra bits \\
\hline \hline unlimited & 33,87 & 2176384 bits & \\
\hline 180 bytes & 33,82 & 2328976 bits & $9640(6.77 \%)$ \\
\hline 160 bytes & 33,83 & 2338320 bits & $19240(7.44 \%)$ \\
\hline 120 bytes & 33,82 & 2407712 bits & $52776(10.62 \%)$
\end{tabular}

TABLE II

REDUNDANCY INTRODUCED WHEN LIMITING THE SIZE OF NALUS (FIRST 197 FRAMES OF FOREMAN.CIF)

In classical video transmission scheme, each packet of compressed data is protected by a CRC. When the CRC is wrong, an ARQ mechanism is put at work. When the joint decoder presented in Section IV-B is involved, the CRC is bypassed and all packets are fed to the decoder. When the standard hard decoder is used, the presence of a CRC is simulated: when a packet is erroneous, it is not provided to the decoder and error concealment techniques are put at work. This allow a fair comparison between the two schemes. In fact, the H.264 decoder is particularly sensitive to bit errors: it may crash after a single bit error. For the simulations, no channel coding has been involved.

Figure 2 shows the Signal to Noise Ratio (SNR) for the luminance as a function of the frame number, for a channel SNR of $11 \mathrm{~dB}$. The performance increase provided by the joint decoder is more than $5 \mathrm{~dB}$ when compared to the hard decoder. Increasing $M$ (the number of simultaneous paths processed by the MA) from 3 to 10 improves the performance of the decoder (with a decoding complexity that is also increased).

Fig. 2. SNR of the luminance, for a channel SNR of $11 \mathrm{~dB}$

Figure 3 represents the average SNR for the luminance of the decoded video as function of the SNR on the channel for the hard decoder (standard solution) and for joint decoders with $M=10$ and $M=3$. At $11 \mathrm{~dB}$, an improvement of more than $5 \mathrm{~dB}$ is obtained by the soft decoder with $M=10$ when compared to the standard solution. The average improvement is more than $4 \mathrm{~dB}$ when $M=3$. When the channel becomes too bad, the error correction capacity of the soft decoding scheme is overflowed and both schemes perform in a similar manner.

The computation complexity of the MA is negligible when compared to that of the H.264 decoder. In optimized implementations, for packets without noise, the decoding complexity of the joint decoding scheme is of the same order of magnitude as that of the standard H.264 decoder. For noisy packets, it is always less than $M$ times the decoding complexity of a standard H.264 decoder. $M$ thus allows to tune some performance-complexity trade off.

Fig. 3. SNR of the luminance as a function of the channel SNR, for NALUs limited to 120 bytes

Figures 4 and 5 show the qualitative improvement brought by the proposed technique. An AWGN channel with an SNR of $11 \mathrm{~dB}$, is considered. Frame number 86 of the decoded sequence using the hard decoder (Figure 4) and the joint decoder with $M=10$ (Figure 5) are both displayed.

Fig. 4. Picture 86 of foreman. cif sent over an AWGN channel with $\mathrm{SNR}=11 \mathrm{~dB}$; NALUs decoded with the standard hard decoder

Fig. 5. Picture 86 of foreman. cif sent over an AWGN channel with $\mathrm{SNR}=11 \mathrm{~dB}$; NALUs decoded with the joint source-channel decoder $(M=$ 10)

\section{CONCLUSIONS AND FUTURE WORK}

The H.264 video coder has been mainly designed to resist to packet losses [3] and shows poor performance when it has to decode packets corrupted with transmission errors. In a wireless environment, with poor channel conditions, obtaining a moderate proportion of lost packets requires strong channel coding. This reduces significantly the usable bandwidth.

This paper provides a joint source-channel decoding technique allowing to partly mitigate the effect of packet transmission errors. Very limited redundancy is introduced, as our joint decoder uses the residual redundancy left in the bitstream by the H.264 video coder. This technique allows less redundant channel codes to be employed, and thus, results in an increase of the average video quality.

Current work is dedicated to further improvements of the joint source-channel decoding technique. For the time being, the tests presented in Section IV-A allow to decide whether a NALU complies with the H.264 syntax. It would be more interesting to determine the origin of the syntax error, which may be due to a transmission error occurring much earlier than the bit position its effect has been detected. An error cannot be detected directly: only its consequences may be detected, which may take some time to appear. A possible way to solve this problem is by exploiting the residual redundancy left by the binarization module of the CABAC.

The optimal redundancy introduction in such video transmission schemes is another important issue that has still to be solved: one has to find the trade off between redundancy introduced at the application level and redundancy at lower levels.

\section{ACKNOWLEDGMENT}

This work has been partly supported by the NEWCOM NoE and by the CNES, contract SDMB. 


\section{REFERENCES}

[1] ITU-T and ISO/IEC JTC 1, "Advanced video coding for generic audiovisual services," Tech. Rep., ITU-T Rec. H.264, and ISO/IEC 14496-10 AVC, nov. 2003.

[2] I. E. G. Richardson, H.264 and MPEG-4 Video Compression: Video Coding for Next Generation Multimedia, Chichester, West Sussex, UK: John Wiley \& Sons, 2003.

[3] T. Stockhammer, M. Hannuksela, and T. Wiegand, "H.264/AVC in wireless environments," IEEE Trans. Circuits and Systems for Video Technology, vol. 13, no. 7, pp. 657-673, 2003.

[4] G. Gennari and G. Mian, "A H.264 robust decoder with error concealment capabilities," ST Journal of Research, vol. 2, no. 1, pp. 67-82, 2005.

[5] A. Kopansky and M. Bystrom, "Sequential decoding of MPEG-4 coded bitstreams for error resilience," in Proc. 33rd Annual Conference on Information Sciences and Systems, 1999.

[6] C. Lamy and O. Pothier, "Reduced complexity maximum a posteriori decoding of variable-length codes," in Proceedings of the IEEE Global Telecommunications Conference, San Antonio, USA, 2001, pp. 14101413.

[7] Q. Chen and K.P. Subbalakshmi, "Joint source-channel decoding for MPEG-4 video transmission over wireless channels," IEEE Journal on Selected Areas in Communications, vol. 21, no. 10, pp. 1780-1789, 2003.

[8] H. Nguyen and P. Duhamel, "Iterative joint source-channel decoding of variable length encoded video sequences exploiting source semantics," in Proceedings of ICIP, 2004.

[9] C.M. Lee, M. Kieffer, and P. Duhamel, "Soft decoding of VLC encoded data for robust transmission of packetized video," in Proceedings of ICASSP, 2005, pp. 737-740.

[10] C. Guillemot and P. Siohan, "Joint source-channel decoding with soft information: A survey," Elsevier Journal on Applied Signal Processing, special issue on the turbo principle, vol. 6, pp. 906-927, 2005.

[11] ITU-T, "Video coding for low bit rate communication," Tech. Rep., ITU-T Rec. H.263, version 1, nov. 1995.

[12] ISO/IEC JTC 1, "Coding of audio-visual objects - part 2: Video," Tech. Rep., ISO/IEC 14496-2 (MPEG-4 visual version 1), apr. 1999.

[13] M. Detlev, H. Schwarz, and T. Wiegand, "Context-based adaptive binary arithmetic coding in the H.264/AVC video compression standard," IEEE Trans. Circuits and Systems for Video Technology, vol. 13, no. 7, pp. 620-636, 2003.

[14] P. G. Howard and J. S. Vitter, "Practical implementations of arithmetic coding," Image and Text Compression, vol. 13, no. 7, pp. 85-112, 1992

[15] S. Saponara, C. Blanch, K. Denolf, and J. Bormans, "The JVT advanced video coding standard complexity and performances analysis on a toolby-tool basis," IEEE Packet Video, pp. 98-109, 2003.

[16] J. B. Anderson and S. Mohan, Source and channel coding: an algorithmic approach, Kluwer Academic Publishers, Norwell, MA, 1991.

[17] ISO/IEC MPEG and ITU-T VCEG Joint Video Team, JM10.2 Test Model CODEC, 2005. 